\title{
NATURAL DIFFERENTIAL OPERATORS ON RIEMANNIAN MANIFOLDS AND REPRESENTATIONS OF THE ORTHOGONAL AND SPECIAL ORTHOGONAL GROUPS
}

\author{
PETER STREDDER
}

In his paper "The foundation of the general theory of relativity", [2], published in 1916, A. Einstein remarked that on a Lorentz manifold $(M, g)$ the only covariant tensors of order 2 which depend in any local coordinate system only on the metric tensor and its first two derivatives, and which depend linearly on the second derivative, are linear combinations of the tensors $g R$ and $R_{i j} d x^{i} \otimes d x^{j}$ where $R$ is the scalar curvature and $R_{i j} d x^{i} \otimes d x^{j}$ the Ricci curvature. In an appendix to [10] H. Weyl proved that $R$ is the only function with these properties.

More recently in [6], P. Gilkey investigated, in a similar vein, forms on Riemannian manifolds and his results are important tools in the proof of the index theorem given by Atiyah, Bott and Patodi in [2]. In [4] D. B. A. Epstein introduces the concept of natural tensor field on Riemannian manifolds. His paper was a major catalyst in the production of this one and should preferably be read before it.

The purpose of this paper is twofold. Firstly it is to study natural tensor fields on Riemannian and oriented Riemannian manifolds. Maintaining the spirit of the earlier results we shall impose a regularity condition on natural tensor fields, which leads to their complete classification as a space of homomorphisms, between certain representation spaces for the general linear group, which are equivariant under the action of the orthogonal or special orthogonal group. The second reason for writing this paper is to define and investigate the notion of natural differential operator in an analogous fashion. It turns out that this problem reduces to the study of natural tensor fields.

We shall only give results on Riemannian manifolds. However P. Gilkey has now extended the Gilkey theorem (cf. [2, \& 2]) to apply to manifolds with an indefinite metric, [8].

The author is indebted to his supervisor, Professor D. B. A. Epstein, who suggested the idea of natural differential operator and who has given him much painstaking advice. The results in $\S 2$ were initiated by a suggestion of M. S.

Received June 13, 1974, and, in revised form, February 5, 1975. 
Narasimhan and have profited from discussions the author has had with him. Thanks are also due to R. Carter and J. C. Kelley for their tuition and advice on representation theory and to N.R. O'Brian for his help with algebraic geometry.

All manifolds, all functions between manifolds and all tensor fields in this paper are $C^{\infty}$.

\section{Preliminaries}

1.1. We shall be concerned with functors $E$ from the category of vector spaces and homomorphisms to itself (see e.g. [5]). With such a functor we shall assume given :

(i) a monomorphism of functors $i_{E}: E \rightarrow T^{r}$ where $T^{r}$ is the $r$ th tensor power functor for some $r$.

(ii) an ordered basis $E\left(v_{i}\right)$ of $E$ for each ordered basis $\left(v_{i}\right)$ of a vector space $V$,

(iii) $E\left(\varphi v_{i}\right)=E \varphi\left(E\left(v_{i}\right)\right)$ for vector spaces $V, W$ and an isomorphism $\varphi \in \operatorname{Hom}(V, W)$.

The rank of $E$ is $r$.

1.2. An inner product $b$ on a vector space $V$ induces an inner product $b$ on $T^{r} V$ and hence on $E V$, which we denote by $E b$. Thus $E V$ is a representation space for $G L(V)$ and $0(V, b)$ with

$$
E(0(V, b)) \subseteq 0(E V, E b)
$$

We denote $(E V)^{*}$ by $E^{*} V$; then $G L(V)$ acts on $E^{*} V$ via $\varphi \mapsto\left(E \varphi^{-1}\right)^{*}$, for $\varphi \in G L(V)$. With this action $0(V, b)$ acts on $E^{*} V$ as a subgroup of $O\left(E^{*} V, E^{*}, b\right)$.

If $\left(v_{i}\right)$ is an ordered basis of $V$ and $E\left(v_{i}\right)=\left(w_{j}\right)$, define the ordered basis $E\left(v^{i}\right)$ of $E^{*} V$ to be the ordered basis $\left(w^{k}\right)$ where $w^{k}\left(w_{j}\right)=\delta_{j}^{k}$.

1.3. Given a Riemannian manifold $(M, g)$, a functor $E$ as in $\S 1.1$ induces Riemannian vector bundles $(E M, E g),\left(E^{*} M, E^{*} g\right)$ over $M$ with connection induced from the Levi-Civita connection. These constructions are functorial and determine subfunctors of the $r$ th tensor power of the tangent bundle and cotangent bundle respectively.

Further it follows from $\S 1.1$ (ii) that given any local coordinate system $x$, there are determined unique ordered local bases of sections $E\left(\partial / \partial x^{i}\right), E\left(d x^{i}\right)$ for $E M, E^{*} M$ respectively.

1.4. We describe in detail certain functors with the properties required in $\S 1.1$, which will be needed later.

Given a vector space $V, S_{r}$ (symmetric group of degree $r$ ) acts on $T^{r} V$ in the usual way.

(i) The functor $S^{r}$.

$$
S^{r} V=\left\{v \in T^{r} V \mid v=\sigma v \quad \text { all } \quad \sigma \in S_{r}\right\}
$$


Let $\operatorname{dim} V=n$ and let $\left(v_{i}\right)$ be an ordered basis for $V$. For each $r$-tuple of integers $\left(i_{1}, \cdots, i_{r}\right), 1 \leq i_{1} \leq \cdots \leq i_{r} \leq n$, let $v_{i_{1} \cdots i_{r}}=\Sigma v_{j_{1}} \otimes \cdots \otimes v_{j_{r}}$ where summation takes place over all distinct $r$-tuples $\left(j_{1}, \cdots, j_{r}\right)$ which are rearrangements of $\left(i_{1}, \cdots, i_{r}\right)$. We let $S^{r}\left(v_{i}\right)=\left(v_{i_{1} \cdots i_{r}}\right)$ ordered by lexicographical ordering on $\left(i_{1}, \cdots, i_{r}\right)$.

(ii) The functors $Y_{r}, r \geq 2$.

Let $T$ be the Young tableau with $r$ squares in the first row and two in the second. Let the first $r+2$ positive integers (starting at 1 ) be arranged in $T$ in increasing order down the columns from left to right. Let $I_{k}$ denote the $k$ th column in this arrangement. For each integer $m$ in $I_{k+1}$ let $P_{k, m}$ denote the set of permutations $\sigma \in S_{r+2}$ which fix exery integer except those in $I_{k} \cup\{m\}$, and which preserve the order of those in $I_{k}$.

$$
\begin{aligned}
Y_{r} V=\left\{v \in T^{r+2} V \mid \sum_{\sigma \in P_{k, m}} \varepsilon(\sigma) \sigma v\right. & =0,1 \leq k \leq r-1, \\
& \left.m \in I_{k+1}, v+(i, j) v=0, i, j \in I_{k}\right\} .
\end{aligned}
$$

For each ordered $(r+2)$-tuple of integers $\left(i_{1}, \cdots, i_{r+2}\right)$ with $1 \leq i_{k} \leq n, i_{1}<i_{2}$; $i_{3}<i_{4} ; i_{1} \leq i_{3} \leq i_{5} \leq i_{6} \leq \cdots \leq i_{r+2} ; i_{2} \leq i_{4}$ let

$$
\begin{aligned}
v_{i_{1} \cdots i_{r}}= & \sum_{\sigma}\left(v_{i_{\sigma(1)}} \otimes v_{i_{\sigma(2)}}-v_{i_{\sigma(2)}} \otimes v_{i_{\sigma(1)}}\right) \\
& \otimes\left(v_{i_{\sigma(3)}} \otimes v_{i_{\sigma(4)}}-v_{i_{\sigma(4)}} \otimes v_{i_{\sigma(3)}}\right) \otimes v_{i_{\sigma(5)}} \otimes \cdots \otimes v_{i_{\sigma(r+2)}}
\end{aligned}
$$

where $\sigma$ runs over all permutations in $S_{r+2}$ which preserve the sets $\{2,4\}$, $\{1,3,5,6, \cdots, r+2\}$ and lead to distinct $(r+2)$-tuples $\left(i_{\sigma(1)}, \cdots, i_{\sigma(r+2)}\right)$. Then $Y_{r}\left(v_{i}\right)=\left(v_{i_{1} \cdots i_{r+2}}\right)$ ordered by lexicographical ordering on $\left(i_{1}, \cdots, i_{r+2}\right)$.

(iii) If $E_{1}, E_{2}$ are functors as in $\S 1.1$, so is $E_{1} \otimes E_{2}$ with lexicographical ordering taken for the basis.

1.5. It is well known that for any vector space $V, Y_{r} V$ is an irreducible representation space for $G L(V)$. For each $r \geq 2$ define $G L(V)$ maps

$$
\alpha_{r}: S^{2}(V) \otimes S^{r}(V) \rightarrow Y_{r}(V) \quad \text { and } \quad \beta_{r}: Y_{r}(V) \rightarrow S^{2}(V) \otimes S^{r}(V)
$$

by

$$
\begin{aligned}
& \alpha_{r}\left(g_{i_{1} \cdots i_{r+2}} v_{i_{1}} \otimes \cdots \otimes v_{i_{r+2}}\right)=\left(g_{i_{1} i_{4} i_{3} i_{2} i_{5} \cdots i_{r+2}}-g_{i_{1} i_{3} i_{2} i_{4} i_{5} \cdots i_{r+2}}\right. \\
& \left.\quad-g_{i_{4} i_{2} i_{3} i_{1} i_{5} \cdots i_{r+2}}+g_{i_{3} i_{2} i_{1} i_{4} i_{5} \cdots i_{r+2}}\right) v_{i_{1}} \otimes \cdots \otimes v_{i_{r+2}}, \\
& \beta_{r}\left(R_{i_{1} \cdots i_{r+2}} v_{i_{1}} \otimes \cdots \otimes v_{i_{r+2}}\right) \\
& =-\frac{r-1}{(r+1) !} \sum_{\sigma} R_{i_{1} i_{\sigma(3)} i_{2} i_{\sigma(4)} i_{\sigma(5)} \cdots i_{\sigma(r+2)}} v_{i_{1}} \otimes \cdots \otimes v_{i_{r}},
\end{aligned}
$$

where $\left(v_{i}\right)$ is an ordered basis of $V$, the summation convention is used, and and sum runs over all permutations $\sigma$ of $\{3, \cdots, r+2\} . \alpha_{r}$ and $\beta_{r}$ do not depend on the basis $\left(v_{i}\right)$ chosen. 
Note that $\alpha_{r}$ and $\beta_{r}$ satisfy:

a) $\alpha_{r} \beta_{r}=\mathrm{id}$,

b) if $\sum_{\sigma \in S_{r+1}} g_{i_{1} i_{\sigma(2)} \cdots i_{\sigma(r+2)}}=0$, where $S_{r+1}$ is the group of permutations of $\{2, \cdots, r+2\}$, then

$$
\beta_{r} \alpha_{r}\left(g_{i_{1} \cdots i_{r+2}} v_{i_{1}} \otimes \cdots \otimes v_{i_{r+2}}\right)=g_{i_{1} \cdots i_{r+2}} v_{i_{1}} \otimes \cdots \otimes v_{i_{r+2}} .
$$

Note that every element in the image of $\beta_{r}$ satisfies this symmetry condition.

1.6. The maps $\alpha_{r}, \beta_{r}$ in $\S 1.5$ determine $G L(V)$ maps $\alpha: \underset{r \geq 2}{\oplus} S^{2}(V) \otimes S^{r}(V)$ $\rightarrow \bigoplus_{r \geq 2} Y_{r}(V)$ and $\beta: \bigoplus_{r \geq 2} Y_{r}(V) \rightarrow \bigoplus_{r \geq 2} S^{2}(V) \otimes S^{r}(V)$ such that $\alpha \beta=$ id. Let $\left(e_{i}\right)$ be the standard basis of $R^{n}$, where $R$ denotes the set of real numbers, with dual basis $\left(e^{i}\right)$. Let $W \in \underset{r \geq 2}{\oplus} Y_{r}^{*} R^{n}$ and suppose that the component of $\beta(W)$ in $S^{2 *} R^{n}$ $\otimes S^{r *} R^{n}$ is the tensor $g_{i_{1} \cdots i_{r+2}} e^{i_{1}} \otimes \cdots \otimes e^{i_{r+2}}$. Let $g(W)_{i j}$ be the real valued functions on $R^{n}(1 \leq i, j \leq n)$ defined by $g(W)_{i j}(x)=\delta_{i j}+\sum_{r=2}^{\infty} g_{i j k_{1} \cdots k_{r}} x^{k_{1}} \ldots$ $x^{k_{r}}$, the superscripts denoting coordinates and not powers.

It follows from $\S 2$ of [4] that these functions determine a Riemannian metric on a neighborhood $U(W)$ of 0 in $R^{n}$. The oriented Riemannian manifold $(U(W), g(W))$ has the inclusion chart as a normal coordinate chart at the origin. It is oriented by the usual orientation on $R^{n}$.

Conversely it is also shown in $\S 2$ of [3] that given a Riemannian manifold $(M, g)$ there exist tensors $W^{r} \in Y_{r}^{*} M(r \geq 2)$, obtained from contracting tensor products of no higher than the $(r-2)$ th covariant derivitive of the curvature tensor, such that in any normal coordinate system at $p \in M$ the coefficients of $\beta_{r}\left(W^{r}(p)\right)$ are the $r$ th partial derivatives of the metric.

\section{The classification theorem}

2.1. Natural tensor fields on Riemannian manifolds are introduced by D. B. A. Epstein in [4]. We extend the definition to the case of oriented Riemannian manifolds.

Definition. Let $E, F$ be functors as in $\S 1.1$. A natural tensor field $t$ on Riemannian manifolds (respectively oriented Riemannian manifolds) of type $(E, F)$ assigns to each Riemannian manifold (resp. oriented Riemannian manifold) $(M, g)$ a tensor field

$$
t(M, g) \in C^{\infty}\left(E M \otimes F^{*} M\right)
$$

such that if $f: M^{\prime} \rightarrow M$ is a diffeomorphism (resp. orientation preserving diffeomorphism) onto an open submanifold then

$$
f^{*} t(M, g)=t\left(M^{\prime}, f^{*} g\right) .
$$

2.2. Epstein has pointed out in [4] that the problem of classifying all natural 
tensor fields is a complicated one. However there is a natural concept of regularity for such tensor fields, which was essentially introduced by Atiyah, Bott and Patodi in [2, §2].

A natural tensor field $t$ of type $(E, F)$ on Riemannian (respectively oriented Riemannian) manifolds is regular if for a given Riemannian (resp. oriented Riemannian) manifold $(M, g)$ with a local coordinate chart $x$ on $U \subseteq M$ the coefficients of $t(M, g)$ with respect to the local basis $E_{1} \otimes F_{2}\left(\partial / \partial x^{i} \otimes \bar{d} x^{j}\right)$ are given by universal polynomials in $g_{i j}, \partial^{|\alpha|} g_{i j} / \partial x^{\alpha}(\alpha$ a multi-index, $|\alpha| \leq N$ large) and $\left(\operatorname{det} g_{i j}\right)^{-1}$ (or (det $\left.g_{i j}\right)^{-\frac{1}{2}}$ in the oriented case). A justification of this definition is given by Atiyah, Bott and Patodi in [2, § 2] for the unoriented case.

On the other hand, the space of oriented Riemannian structures on a vector space $V$ is naturally identified with $G L(n, R) / S O(n, R), n=\operatorname{dim} V$. It is well known and is shown in the appendix $(A .2)$ that any rational function $f$ on $G L(n, R)$ invariant under the action of $S O(n, R)$ is of form :

$$
f(A)=F\left(A A^{t}\right)+(\operatorname{det} A)^{-1} G\left(A A^{t}\right), \quad A \in G L(n, R),
$$

where $F, G: S M(n, R) \rightarrow R$ are rational functions on the space of symmetric matrices.

Since the identification of $G L(n, R) / S O(n, R)$ with the space of oriented Riemannian structures is given by

$$
[A] \in G L(n, R) / S O[n, R) \rightarrow\left(A A^{t}, \operatorname{sign}(\operatorname{det} A)\right),
$$

the corresponding identification of rings of rational functions shows that it natural to regard $\left.R\left[g_{i j} \text {, (det } g_{i j}\right)^{-\frac{1}{2}}\right]$ as the ring of functions on the space of oriented Riemannian structures.

Remark. In applications to the index theorem, polynomial dependence on $\left(\operatorname{det} g_{i j}\right)^{-\frac{1}{2}}$ appears explicity even in the unoriented case although this was overlooked in the original proof in [2]; see [1]. In fact, it follows from [4, Theorem 5.2] that even if we merely demand that the coefficients of our tensor field be given by universal polynomials in $\partial g_{i j} / \partial x^{\alpha}(\alpha$ a multi-index, $1 \leq|\alpha| \leq N$ large) with coefficients functions of the $g_{i j}$ (not necessarily continuous) then the tensor field is regular (polynomial in the terminology of [4]).

2.3. An important class of natural tensor fields is those which are homogeneous (see [4, $\S \S 6 \& 7]$ ). A natural tensor field $t$ is homogeneous of weight $k$ if $t\left(M, \lambda^{2} g\right)=\lambda^{k} t(M, g)$ for all real numbers $\lambda$. Note that $g$ is homogeneous of weight 2 and that the tensors $W^{r}(r \geq 2)$ introduced in $\S 1.3$ are also homogeneous of weight 2 . If $t$ has weight $k$ and is of type $(E, F)$ with $\operatorname{rank} E=a$ and rank $F=b$, then $t$ has normalised weight $w=b-a-k . t$ has maximal weight if $w=0$.

2.4. Before proceeding with the main theorem, we need the following crucial lemma.

Lemma. Let $(V,\langle\rangle$,$) be an oriented inner product space.$ 
(i) The vector space $\operatorname{Hom}_{0(V)}\left(\otimes^{k} V, R\right)$ is zero for odd $k$, and for even $k$ it is spanned by elements of the form

$$
v_{1} \otimes \cdots \otimes v_{k} \mapsto\left\langle v_{\pi(1)}, v_{\pi(2)}\right\rangle \cdots\left\langle v_{\pi(k-1)}, v_{\pi(k)}\right\rangle,
$$

where $\Pi \in S_{k}$.

(ii) The vector space $\operatorname{Hom}_{S O(V)}\left(\stackrel{k}{\otimes}^{2} V, R\right)$ is equal to $\operatorname{Hom}_{O(V)}(\stackrel{k}{\otimes} V, R)$ except that if $k-n(n=\operatorname{dim} V)$ is nonnegative and even, there is permitted any linear combination of maps

$$
\begin{aligned}
v_{1} \otimes & \cdots \otimes v_{k} \mapsto \\
& \sum_{\mu \in S_{n}} \varepsilon(\mu) v_{\pi(1) \mu(1)} \cdots v_{\pi(n) \mu(n)}\left\langle v_{\pi(n+1)}, v_{\pi(n+2)}\right\rangle \cdots\left\langle v_{\pi(k-1)}, v_{\pi(k)}\right\rangle,
\end{aligned}
$$

where $\pi \in S_{k}$, and $v_{j}=\sum_{l=1}^{n} v_{j_{1}} e_{1},\left(e_{1}, \cdots, e_{n}\right)$ being a positively oriented basis.

Proof. (i) is proved in [2, Appendix 1].

(ii) is proved in $[11$, p. 64$]$; a proof is also given in the appendix $\S 5.1$ to this paper.

2.5. The theorem we shall prove in this section tells us that every natural regular tensor field on Riemannian manifolds is polynomial in the sense of Epstein $[4, \S 5]$. However it goes further than this. It follows from the theorem and the theory of representations that the space of homogeneous natural regular tensor fields of some fixed weight is finite dimensional and that the problem of calculating it reduces to a problem in representation theory.

In the oriented case, in addition to the usual polynomial tensor fields, there are allowed tensor fields of the form:

$$
\sum_{\pi \in S_{n}}\left(\operatorname{det} g_{i j}\right)^{\frac{1}{2}} g^{\pi(1)} \ldots g^{\pi(n)} \cdot g^{*} \ldots g \cdot g_{. .} \cdots g . . W_{. .}^{\varepsilon_{1}} \ldots W_{. .}^{\varepsilon r} .
$$

where the dots indicate contraction or summation with a local basis.

Equivalently we introduce tensor fields of the form :

$$
\sum_{\pi \in S_{n}}\left(\operatorname{det} g_{i j}\right)^{\frac{1}{2}} g^{\pi(1)} \cdots g^{\pi(n)} \cdot g^{(1)} \ldots g \cdot g_{. .} \cdots g_{. .} \nabla R_{. .}^{\varepsilon_{1}} \cdots \nabla R_{. .}^{\varepsilon_{r}},
$$

where $R$ is the Riemann-Christoffel tensor $R_{i j k l} d x^{i} \otimes d x^{j} \otimes d x^{k} \otimes d x^{l}$.

Theorem. Let $R$ denote the set of real numbers. Then there is a bijection between natural regular tensor fields on Riemannian manifolds (respectively oriented Riemannian manifolds) of type $(E, F)(\operatorname{rank} E=a$, rank $F=b)$ and equivariant $O(n)$ (resp. $S O(n))$ homomorphisms:

$$
\begin{aligned}
& \varphi: R \oplus \bigoplus_{i=1}^{\infty} \underset{2 \leq r_{1}<\cdots<r_{i}}{\bigoplus} \bigoplus_{s=1}^{\infty} \underset{\substack{j_{1} \\
j_{1}+\cdots, j_{i}>s}}{\bigoplus} S_{j_{i}=s}^{j_{1}}\left(Y_{r_{i}}{ }^{*} R^{n}\right) \otimes \cdots \otimes S^{j_{i}}\left(Y_{r_{i}}{ }^{*} R^{n}\right) \\
& \rightarrow E R^{n} \otimes F^{*} R^{n}
\end{aligned}
$$


which vanish except on a finite number of direct summands. Further:

(i) There are no such tensor fields, homogeneous of normalised weight $w<0$, or $w=1$.

(ii) The tensor filds which are homogeneous of maximal weight correspond bijectively to $O(n)$ (resp. $S O(n))$ maps

$$
\varphi: R \rightarrow E R^{n} \otimes F^{*} R^{n} .
$$

(iii) The tensor fields, homogeneous of normalised weight $w \geq 2$, correspond bijectively to $O(n)$ (respectively $S O(n)$ ) maps

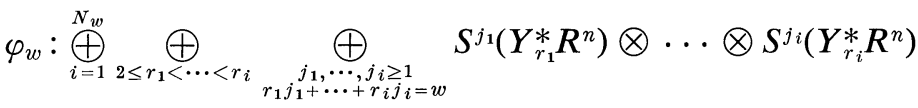

$$
\begin{aligned}
& \rightarrow E R^{n} \otimes F^{*} R^{n},
\end{aligned}
$$

where $N_{w}=\left[\frac{1}{2}\left(-3+(9+8 w)^{\frac{1}{2}}\right)\right]$.

Proof. The proof is given in the oriented case. The unoriented case is slightly simpler. So let $t$ be a natural regular tensor field on oriented Riemannian manifolds. Define $\psi_{t}: R \oplus \bigoplus_{r \geq 2} Y_{r}^{*} R^{n} \rightarrow E R^{n} \otimes F^{*} R^{n}$ by $W \mapsto t(U(W), g(W))(O)$ identifying the fibre of $E U(W) \otimes F^{*} U(W)$ at $O$ with $E R^{n} \otimes F^{*} R^{n}$ via the canonical basis determined by the inclusion chart.

Now let $a \in S O(n)$. Then the expansion of $g_{i j}(a W)$ at $O$ in the normal coordinate chart determined by $a$ is the same as that of $g_{i j}(W)$ with respect to the inclusion chart. Since the coefficients of $t$ are given by universal polynomials, the coefficients of $t(U(a W), g(a W))(O)$ with respect to the basis of $E R^{n} \otimes F^{*} R^{n}$ obtained by applying $a$ to the standard basis are the same as those of $t(U(W)$, $g(W))(O)$ with respect to the standard basis. Thus $\psi_{t}$ is an equivariant polynomial map vanishing except on a finite number of direct summands. Complete polarisation determines $\varphi_{t}$.

Conversely, suppose an equivariant $S O(n)$ map $\varphi$ is given. Now let $(M, g)$ be an oriented Riemannian manifold, and let $p \in M$. Then there is a natural identification of $T_{p} M$ with $R^{n}$ which is well defined up to composition with elements of $S O(n)$. Since $\varphi$ is equivariant under the action of $S O(n), \varphi$ determines a unique $S O\left(T_{p} M, g(p)\right)$ map

$$
\begin{gathered}
\varphi(M, g)(p): R \oplus \bigoplus_{i=2}^{\infty} \underset{2 \leq r_{1}<\cdots<r_{i}}{\oplus} \bigoplus_{s=1}^{\infty} \underset{\substack{j_{1}, \cdots, j_{i} \geq 1 \\
j_{1}+\cdots+j_{i}=s}}{\bigoplus_{p}} S_{p}^{j_{1}}\left(Y_{r_{1}}^{*} M\right) \otimes \cdots \otimes S_{p}^{j_{i}}\left(Y_{r_{i}}^{*} M\right) \\
\rightarrow E_{p} M \otimes F_{p}^{*} M,
\end{gathered}
$$

vanishing except on a finite number of summands. Define

$$
\begin{aligned}
& t_{\varphi}(M, g)(p) \\
& \quad=\varphi(M, g)(p)\left(1 \oplus \bigoplus_{i=2}^{N} \underset{2 \leq r_{1}<\cdots<r_{i}}{\bigoplus} \bigoplus_{s=1}^{\infty} \underset{\substack{j_{1}, \ldots, j_{i} \geq 1 \\
j_{1}+\cdots+j_{i}=s}}{\bigoplus^{\prime}} W^{r_{1}}(p)^{j_{1}} \otimes \cdots \otimes W^{r_{i}}(p)^{j_{i}}\right)
\end{aligned}
$$


with $N$ large.

If follows from $\S 2.4$ that $t_{\varphi}$ is determined in the required way by universal polynomials. Since the whole construction is functorial, $t_{\varphi}$ is the required natural tensor field. Clearly $\varphi_{t \varphi}=\varphi$. Conversely it follows from $\S 1.5$ and $\S 2.4$ that $t_{\varphi_{t}}=t$.

For that last part of the theorem consider $S O(n)$ maps :

$$
S^{j_{1}}\left(Y_{r_{1}}^{*} R^{n}\right) \otimes \cdots \otimes S^{j_{i}}\left(Y_{r_{i}}^{*} R^{n}\right) \rightarrow E R^{n} \otimes F^{*} R^{n},
$$

with $j_{k} \geq 1,2 \leq r_{1}<\ldots<r_{i}$. These are determined by $S O(n)$ maps :

$$
S^{j_{1}}\left(Y_{r_{1}}^{*} R^{n}\right) \otimes \cdots \otimes S^{j_{i}}\left(Y_{r_{i}}^{*} R^{n}\right) \otimes \bigotimes^{a} R^{n *} \otimes \bigotimes^{b} R^{n} \rightarrow R,
$$

and hence the component natural tensor fields thus obtained are of two types:

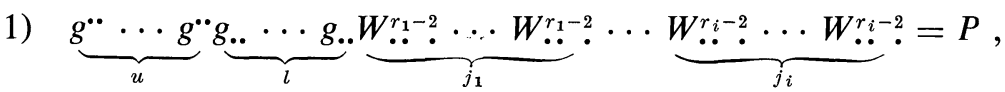

where there are $c$ contractions and summation is over all indices except $a$ upper and $b$ lower.

2) $\sum_{\pi \in S_{n}}\left(\operatorname{det} g_{i j}\right)^{\frac{1}{2}} g^{\pi(1)} \cdot \cdots g^{\pi(n)} \cdot P$,

where there are $c$ contractions and summation is over all indices except $a$ upper and $b$ lower.

In case 1 ), equating weights gives $b-a-w=-2 u+2 l+2 \sum_{k=1}^{i} j_{k}$, counting indices gives $a=2 u-c, b=2 l+\sum_{k=1}^{i} r_{k} j_{k}+2 \sum_{k=1}^{i} j_{k}-c$, whence $w=$ $\sum_{k=1}^{i} r_{k} j_{k}$

In case 2), equating weights gives $b-a-w=n+2 l+2 \sum_{k=1}^{i} j_{k}-2 u-$ $2 n$, counting indices gives $a=2 u+n-c, b=2 l+\sum_{k=1}^{i} r_{k} j_{k}+2 \sum_{k=1}^{i} j_{k}-c$, whence $w=\sum_{k=1}^{i} r_{k} j_{k}$.

That $w$ cannot equal 1 follows from $r_{k} \geq 2$ for all $k$. Finally the computation of $N_{w}$ is left to the reader.

Remark. For future reference we note that in the unoriented case all natural regular homogenous tensor fields have even weight.

2.6. Finally in this section we extend the Gilkey theorem [2, § 2] to the oriented case.

Recall that $*: C^{\infty}\left(\stackrel{r}{\wedge} T^{*} M\right) \rightarrow C^{\infty}\left(\bigwedge^{n-r} T^{*} M\right)$ is defined by $\omega^{\prime} \wedge * \omega=\left\langle\omega^{\prime}\right.$, 
$\omega>v$, where $\left(M^{n}, g\right)$ is an oriented Riemannian manifold, $\omega^{\prime}$ is any $r$-form, and $v$ is the orientation form given in a positively oriented local coordinate system by $\left(\text { det } g_{i j}\right)^{\frac{1}{2}} d x^{1} \wedge \cdots \wedge d x^{n}$. Further, $*$ maps natural regular $r$-forms on oriented Riemannian $n$-manifolds to natural regular $(n-r)$-forms on oriented Riemannian $n$-manifolds.

Corollary. The natural regular homogeneous $r$-forms on oriented Riemannian manifolds $\left(M^{n}, g\right)$ of weight $k$ are linear combinations of forms of two types:

1) Natural regular r-forms $\omega$ on Riemannian manifolds, homogeneous of weight $k$. For $k=0$ these are precisely the Pontrjagin r-forms.

2) The forms $* \omega$ where $\omega$ is a natural regular $(n-r)$-form on Riemannian manifolds, homogeneous of weight $k+n-2 r$.

In particular the conformal (weight 0 ) $n$-forms are sums of:

a) The Pontrjagin $n$-forms,

b) The forms $f\left(\operatorname{det} g_{i j}\right)^{\frac{1}{2}} d x^{1} \wedge \cdots \wedge d x^{n}$ where $f$ is a natural regular function on Riemannian manifolds, homogeneous of weight $-n$.

Thus if $n$ is odd, it follows from the remark of $\S 2.5$ that there are no conformal natural regular $n$-forms.

Proof. It follows from [2, § 2], § 2.5 above and the fact that $*$ adds $n-2 r$ to the weight of a homogeneous $r$-form.

Remark. I. M. Singer has conjectured that if $\omega$ is a natural regular $n$-form on oriented Riemannian $n$-manifolds and $I(M)=\int_{M} \omega(M, g)$ is independent of the metric for each $n$-manifold $M$, then there are a real number $c$, a natural regular $(n-1)$-form $\rho$ and a Pontrjagin $n$-form $\eta$ such that $\omega=d \rho+c E_{n}+\eta$ where $E_{n}$ is the Euler class. Certainly $\omega$ has to be conformal, for if we write $\omega=\sum_{i \geq 0} \omega_{i}$ where $\omega_{i}$ is homogeneous of weight $i$, then for all real numbers $\lambda$

$$
I(M)=\sum_{i \geq 0} \int_{M} \omega_{i}(M, g)=\sum_{i \geq 0} \int_{M} \omega_{i}(M, \lambda g)=\sum_{i \geq 0} \lambda^{i} \int_{M} \omega_{i}(M, g) .
$$

Hence $\int_{M} \omega_{i}(M, g)=0, i>0$, and $\omega$ has to be of type a) or b). The conjecture has now been confirmed by P. Gilkey [7].

\section{Natural differential operators}

3.1. For a review of differential operators, we refer the reader to R. S. Palais [9]. Before making our definitions however, there are some notions which we would like to recall explicitly.

3.2. Let $\xi, \eta$ be $C^{\infty}$ vector bundles over a smooth manifold $M$, with $C^{\infty}(\xi)$ the space of $C^{\infty}$ sections of $\xi$. We let $\operatorname{Diff}_{k}(\xi, \eta)$ denote the space of differential operators of order $\leq k$ from $C^{\infty}(\xi)$ to $C^{\infty}(\eta)$. Let $S^{k}(\xi)$ denote the $k$-fold sym- 
metric tensor power of $\xi$ with itself, and let $S^{k}:{ }^{k} \xi \rightarrow S^{k}(\xi)$ be the map characterized by

$$
S^{k}\left(v_{1} \otimes \cdots \otimes v_{k}\right)=(k !)^{-1} \sum_{\pi \in S_{k}} v_{\pi(1)} \otimes \cdots \otimes v_{\pi(k)}
$$

where $v_{i} \in \xi_{x}$ for some $x \in M$. Then we have the symbol exact sequence:

$$
0 \longrightarrow \operatorname{Diff}_{k-1}(\xi, \eta) \stackrel{i}{\longrightarrow} \operatorname{Diff}_{k}(\xi, \eta) \stackrel{r_{k}}{\longrightarrow} \operatorname{Hom}\left(S^{k}\left(T^{*} M\right) \otimes \xi, \eta\right) \longrightarrow 0
$$

where $i$ is inclusion and $\gamma_{k}$ is characterized by

$$
\gamma_{k}(D)\left(S^{k}\left(v_{1} \cdots v_{k}\right) \otimes e\right)=(k !)^{-1} D\left(g_{1} \cdots g_{k} s\right)(x)
$$

where $g_{i} \in C^{\infty}(M), g_{i}(x)=0, d_{x} g_{i}=v_{i} \in T^{*} M, s \in C^{\infty}(\xi), s(x)=e \in \xi_{x}$.

3.3. Let $E, F$ be functors as in $\S 1.1$. Recall that given a Riemannian manifold $(M, g)$ there is a unique torsion free connection $V$ on $T M$ satisfying $\nabla g=0$. This is the Levi-Civita connection. $\nabla$ induces a connection $\nabla$ on $E M \otimes F^{*} M$ in a natural way.

Define differential operators

$$
D_{k}: C^{\infty}\left(E M \otimes F^{*} M\right) \rightarrow C^{\infty}\left(S^{k}\left(T^{*} M\right) \otimes E M \otimes F^{*} M\right)
$$

by taking the composition:

$$
\begin{aligned}
C^{\infty}\left(E M \otimes F^{*} M\right) & \stackrel{\nabla^{k}}{\longrightarrow} C^{\infty}\left({ }^{k} T^{*} M \otimes E M \otimes F^{*} M\right) \\
& \stackrel{S^{k} \otimes 1}{\longrightarrow} C^{\infty}\left(S^{k}\left(T^{*} M\right) \otimes E M \otimes F^{*} M\right) .
\end{aligned}
$$

Then $\gamma_{k}\left(D_{k}\right) \in \operatorname{Hom}\left(S^{k}\left(T^{*} M\right) \otimes E M \otimes F^{*} M, S^{k}\left(T^{*} M\right) \otimes E M \otimes F^{*} M\right)$ is the identity map.

3.4. Definition. Let $E, F, G, H$, be functors as in $\S 1.1$. A natural differential operator of type $(E, F, G, H)$ on Riemannian (resp. oriented Riemannian) manifolds assigns to each Riemannian (resp. oriented Riemannian) manifold $(M, g)$ a differential operator $D(M, g): E M \otimes F^{*} M \rightarrow G M \otimes H^{*} M$ such that if $f: M \rightarrow M^{\prime}$ is a diffeomorphism onto an open submanifold (resp. orientation preserving diffeomorphism onto an open submanifold) then $D\left(M^{\prime}\right.$, $\left.f^{*} g\right)=f^{*}(D(M, g))$.

3.5. Let $(M, g)$ be a Riemannian manifold and let $x$ be a local coordinate system on $U \subseteq M$. Then $x$ determines local bases of sections $\left(e_{\alpha}\right)_{\alpha \in A},\left(f^{\beta}\right)_{\beta \in B}$, $\left(g_{\gamma}\right)_{\gamma \in C},\left(h^{\delta}\right)_{\delta \in D}$ for $E M, F^{*} M, G M, H^{*} M$ as in $\S 1.3$. Let $D: E M \otimes F^{*} M \rightarrow$ $G M \otimes H^{*} M$ be a differential operator of order $\leq k$. Then locally we may write 


$$
D\left(s_{\beta}^{\alpha} e_{\alpha} \otimes f^{\beta}\right)=\sum_{r=0}^{k} a_{\alpha \delta}^{\beta r i_{1} \cdots i_{r}} \frac{\partial^{r} s_{\beta}^{\alpha}}{\partial x^{i_{1}} \cdots \partial x^{i_{r}}} g_{\gamma} \otimes h^{\delta}
$$

using the summation convention, where the functions $a_{\alpha_{\delta}}^{\beta \gamma i_{1} \cdots i_{r}}$ are symmetric in $i_{1}, \cdots, i_{r}(2 \leq r \leq k)$. We refer to the local functions $a_{\alpha_{\delta}}^{\beta r i_{1} \cdots i_{r}}$ as the coefficients of $D$ with respect to the coordinate system $x$. Note that locally:

$$
\gamma_{k}(D)\left(v_{\beta i_{1} \cdots i_{r}}^{\alpha} d x^{i_{1}} \otimes \cdots \otimes d x^{i_{r}} e_{\alpha} \otimes f^{\beta}\right)=a_{\alpha_{\delta}}^{\beta r i_{1} \cdots i_{r}} v_{\beta i_{1} \cdots i_{r}}^{\alpha} g_{r} \otimes h^{\tilde{o}} .
$$

3.6. A natural differential operator $D$ on Riemannian manifolds (resp. oriented Riemannian manifolds) is regular if the coefficients of $D(M, g)$ in any local coordinate system are given by universal polynomials in $g_{i j}, \partial^{|\alpha|} g_{i j} / \partial x^{\alpha}(\alpha$ a multi-index $|\alpha|<N, N$ large) and $\left(\operatorname{det} g_{i j}\right)^{-1}\left(\operatorname{resp}\right.$. $\left.\left(\operatorname{det} g_{i j}\right)^{-\frac{1}{2}}\right)$. The operators $D_{k}$ introduced in $\S 3.3$ are examples of such operators. Note also that natural bundle maps and natural tensor fields correspond bijectively, and are therefore classified by $\S 2.5$. Our main theorem says that in fact this classification also works for natural differential operators.

3.7. Theorem. Let $D$ be a natural differential operator of type $(E, F, G$, $H$ ) and order $k$. Then there are unique natural bundle maps

$$
t_{r}: C^{\infty}\left(S^{r}\left(T^{*} M\right) \otimes E M \otimes F^{*} M\right) \rightarrow C^{\infty}\left(G M \otimes H^{*} M\right) \quad(0 \leq r \leq k)
$$

such that $D=\sum_{r=0}^{k} t_{r} D_{r}$, and the $t_{r}$ are regular if and only if $D$ is.

Proof. The result is proved by induction on $k$ and is clear for $k=0$. Suppose that the result has been proved for operators of order $k-1$, and let $D$ have order $k$. Then $\gamma_{k}(D)$ is a natural bundle map which is regular if $D$ is and $\gamma_{k}(D) D_{k}$ is a natural differential operator of order $k$. Since $\gamma_{k}\left(D-\gamma_{k}(D) D_{k}\right)=0$ by the remark at the end of $\S 3.3, D-\gamma_{k}(D) D_{k}$ is a natural differential operator of order $k-1$, regular if $D$ is. Setting $t_{k}=\gamma_{k}(D)$, the result follows by induction.

\section{Examples}

4.1. Let $D$ be a natural regular differential operator on Riemannian manifolds of order $k$ and type $(E, F, G, H)$ with the ranks of $E, F, G, H$ equal to $a, b, c, d$ respectively. $D$ is determined by natural regular bundle maps:

$$
t_{r}: C^{\infty}\left(S^{r}\left(T^{*} M\right) \otimes E M \otimes F^{*} M\right) \rightarrow C^{\infty}\left(G M \otimes H^{*} M\right) .
$$

If follows from the general theory in $\S 2.5$ that if $t_{r}$ is homogeneous of weight $w_{r}$ then

1) $w_{r}<a+d-b-c-r$, and further

2) if a monomial appears in $t_{r}$ involving exactly $j_{l}$ terms $W^{\varepsilon}$ (equivalently $\left.\nabla^{\varepsilon_{l}-2} R\right), 1 \leq l \leq i, 2 \leq \varepsilon_{l}<\varepsilon_{l+1}$, then 


$$
a+d=b+c+r+w_{r}+\sum_{l=1}^{i} j_{i} \varepsilon_{i} .
$$

Thus $t_{r}=0$ if $w_{r}>a+d-b-c-r, w_{r}=a+d-b-c-r-1$ or $w_{r}$ odd (by $\S 2.5$ ). We say that $D$ has maximal weight if it is homogeneous of weight $a+d-b-c-k$.

The homogeneous natural regular differential operators of maximal weight are of some interest since any differential operator between vector bundles over $R^{n}$ which is the evaluation of a natural regular operator is a sum of these.

4.2. Hence, if $D$ in $\S 4.1$ has order 1 and maximal weight, then $D=\sigma \circ \nabla$, where $\sigma$ is a natural bundle map and $\nabla$ the Levi-Civita connection. Thus operators of maximal weight and order 1 correspond bijectively with bundle maps. It follows that the Levi-Civita connection on $E M \otimes F^{*} M$ is the unique connection of maximal weight, which in this case is of weight 0 (cf. Epstein [4, Theorem 5.6]).

Similarly the exterior derivative on forms and its adjoint are unique of maximal weight, in this case weights 0 and -2 respectively, up to multiplication by constants.

Finally note that it follows from $\S 4.1$ that there are no natural vector fields homogeneous of weight greater than -4 .

4.3. Having seen that the Levi-Civita connection is unique of maximal weight, we move on to consider the Laplacian on forms. Again we consider the unoriented case. The situation is not as simple as in the order 1 case, but we can say the following.

Let $\sigma_{1}, \sigma_{2}: C^{\infty}\left(\bigwedge^{p} T^{*} M\right) \rightarrow C^{\infty}\left(\bigwedge^{p} T^{*} M\right)$ be the bundle maps defined by :

$$
\begin{aligned}
& \sigma_{1}\left(d x^{i_{1}} \wedge \cdots \wedge d x^{i_{r}}\right)=\sum_{s=1}^{r} \sum_{j=1}^{n} R_{j}^{i_{s}} d x^{i_{1}} \wedge \cdots \wedge d x^{i_{s-1}} \\
& \wedge d x^{j} \wedge d x^{i_{s+1}} \wedge \cdots \wedge d x^{i_{r}} \\
& \sigma_{2}\left(d x^{i_{1}} \wedge \cdots \wedge d x^{i_{r}}\right)=\sum_{1 \leq s<t \leq r} \sum_{j, k=1}^{n} R^{i_{s} i_{t}}{ }_{j k} d x^{i_{1}} \wedge \cdots \wedge d x^{i_{s-1}} \\
& \wedge d x^{j} \wedge d x^{i_{s+1}} \wedge \cdots \wedge d x^{i_{t-1}} \\
& \wedge d x^{k} \wedge d x^{i_{t+1}} \wedge \cdots \wedge d x^{i_{r}},
\end{aligned}
$$

where $R_{i}{ }^{j}$ is the Ricci tensor, $R^{i j}{ }_{k l}$ is the curvature tensor with second index raised and $\operatorname{dim} M=n$. Then $\sigma_{1}$ and $\sigma_{2}$ are self-adjoint. Let $R$ be the scalar curvature, $d$ the exterior derivative, $d^{*}$ its adjoint and $\nabla$ the Laplacian. Let $D: C\left(\bigwedge^{p} T^{*} M\right) \rightarrow C\left(\bigwedge^{p} T^{*} M\right)$ be a natural regular differential operator of maximal weight, -2 in this case, and order 2 . Then :

1) $D=a \Delta+c R$, if $p=0$ or $n$,

2) $D=a_{1} d d^{*}+a_{2} d^{*} d+b \sigma_{1}+c R$, if $p=1$ or $n-1$, 
3) $D=a_{1} d d^{*}+a_{2} d^{*} d+b_{1} \sigma_{1}+b_{2} \sigma_{2}+c R$, if $2 \leq p \leq n-2$, where $a, a_{1}, a_{2}, b, b_{1}, b_{2}, c$ are uniquely determined constants. Further, if $D$ is self-adjoint then $a_{1}=a_{2}=a$ so that :

2) $D=a \Delta+b \sigma_{1}+c R$, if $p=1$ or $n-1$,

3) $D=a \Delta+b_{1} \sigma_{1}+b_{2} \sigma_{2}+c R$, if $2 \leq p \leq n-2$.

\section{Appendix}

5.1. Proof of part (ii) of the lemma in $\S 2.4$ for $V=R^{n}, O(V)=S O_{n}$. Note that $Z_{2}=O_{n} / S O_{n}$ acts on Hom $\left(\otimes^{k} R^{n}, R\right)$ splitting it as the direct sum of $\operatorname{Hom}_{o_{n}}\left(\stackrel{k}{\otimes} R^{n}, R\right)$ and the -1 eigenspace $\wedge$. If $f \in \wedge$, then define $\tilde{f} \in \operatorname{Hom}_{O_{n}}\left(\AA^{n+k} R^{n}, R\right)$ by

$$
\tilde{f}\left(v_{1} \otimes \cdots \otimes v_{k+n}\right)=f\left(v_{1} \otimes \cdots \otimes v_{k}\right) \sum_{\mu \in S_{n}} \varepsilon(\mu) v_{k+1, \mu(1)} \cdots v_{k+n, \mu(n)},
$$

so that $\tilde{f}\left(v_{1} \otimes \cdots \otimes v_{k} \otimes e_{1} \otimes \cdots \otimes e_{n}\right)=f\left(v_{1} \otimes \cdots \otimes v_{k}\right)$. Hence

$$
\begin{gathered}
f\left(v_{1} \otimes \cdots \otimes v_{k}\right)=\sum_{\pi \in S_{k}} c_{\pi} v_{\pi(1) 1} \cdots v_{\pi(n) n}\left\langle v_{\pi(n+1)}, v_{\pi(n+2)}\right\rangle \\
\\
\cdots\left\langle v_{\pi(k-1)}, v_{\pi(k)}\right\rangle
\end{gathered}
$$

for some constants $c_{\pi}$ if $k-n$ is positive and even, and $f\left(v_{1} \otimes \cdots \otimes v_{k}\right)$ is zero otherwise. But $\mu \in S_{n}$ determines an element $\mu \in O_{n}$ of determinant $\varepsilon(\mu)$ by permuting coordinates. Thus

$$
\begin{array}{r}
f\left(v_{1} \otimes \cdots \otimes v_{k}\right)=\varepsilon(\mu) f\left(\mu v_{1} \otimes \cdots \otimes \mu v_{k}\right)=\sum_{\pi \in S_{k}} \varepsilon(\mu) c_{\pi} v_{\pi(1) \mu(1)} \\
\cdots v_{\pi(n) \mu(n)}\left\langle v_{\pi(n+1)}, v_{\pi(n+2)}\right\rangle \cdots\left\langle v_{\pi(k-1)}, v_{\pi(k)}\right\rangle .
\end{array}
$$

Since $f \in \wedge$, we have

$$
\begin{aligned}
& f\left(v_{1} \otimes \cdots \otimes v_{k}\right)=(n !)^{-1} \sum_{\pi \in S_{k}} c_{\pi} \sum_{\mu \in S_{n}} \varepsilon(\mu) v_{\pi(1) \mu(1)} \\
& \cdots v_{\pi(n) \mu(n)}\left\langle v_{\pi(n+1)}, v_{\pi(n+2)}\right\rangle \cdots\left\langle v_{\pi(k-1)}, v_{\pi(k)}\right\rangle
\end{aligned}
$$

if $k-n$ is positive and even, and $f\left(v_{1} \otimes \cdots \otimes v_{k}\right)$ is zero otherwise.

5.2. Lemma. Any rational function $f$ on $G L(n, R)$ invariant under the action of $S O(n, R)$ by right multiplication is of form:

$$
f(A)=F\left(A A^{t}\right)+(\operatorname{det} A)^{-1} G\left(A A^{t}\right), \quad A \in G L(n, R),
$$

where $F, G: S M(n, R) \rightarrow R$ are rational functions on the space of symmetric matrices. 
Proof. Consider the space of rational functions

$$
f: G L(n, R) \rightarrow R
$$

invariant under right multiplication by elements of $S O_{n}$. Again $Z_{2}=O_{n} / S O_{n}$ acts on this space, splitting it as the direct sum of the $O_{n}$ invariant maps and the -1 eigenspace $\wedge$. If $f \in \wedge$, then $h: G L(n, R) \rightarrow R: X \mapsto(\operatorname{det} X) f(X)$ is $O_{n}$ invariant and hence $f(X)=(\operatorname{det} X)^{-1} h(X)$. The required result then follows from Appendix 1 of [2].

\section{References}

[1] M. F. Atiyah, The heat equation in Riemannian geometry, Séminaire Bourbaki, 26ième année, 1973-74, Exp. 436.

[2] M. F. Atiyah, R. Bott \& V. K. Patodi, On the heat equation and the index theorem, Invent. Math. 19 (1973) 279-330.

[3] A. Einstein, The foundation of the general theory of relativity. The principle of relatively, Dover Publications, New York, 1923, 109-264.

[4] D. B. A. Epstein, Natural tensors on Riemannian manifolds, J. Differential Geometry 10 (1975) 631-645.

[ 5 ] D. B. A. Epstein \& M. Kneser, Functors between categories of vector spaces, Lecture Notes in Math. Vol. 99, Springer, Berlin, 1969, 154-170.

[6] P. Gilkey, Curvature and the eigenvalues of the Laplacian for elliptic complexes, Advances in Math. 10 (1973) 344-382.

[ 7 ] - Local invariants of a Riemannian manifold, to appear.

[8] - to appear.

[ 9 ] R. S. Palais, Seminar on the Atiyah-Singer index theorem, Annals of Math. Studies No. 57, Princeton University Press, Princeton, 1965, 27-94.

[10] H. Weyl, Space. Time. Matter, Dover Publications, New York, 1922, Appendix.

[11] —, Classical groups, Princeton University Press, Princeton, 1939.

UNIVERSITY OF WARWICK 